\title{
Understanding Primitive Reflexes and Their Role in Growth and Development: A Review
}

Reflexes are set motor responses to specific sensory stimuli. In newborns and young infants these primitive reflexes are an important assessment tool. Children with a distinctive reflex are difficult to treat. This includes a large category in which primitive reflexes are retained longer than necessary. Certain reflexes may not appear at appropriate age of development. Many neurological conditions are characterized by aberrations in reflex actions. However, there is scarcity of data for high-risk infants pertaining to this topic. Dental treatment becomes challenging in these individuals and sometimes due to lack of compliance even necessary emergency dental treatment is difficult to carry out.

KEYWORDS: Primitive Reflexes, Infantile Reflexes, Growth and Development

\section{INTRODUCTION}

Primitive reflexes can be defined as an automatic movement beginning as early as 25-26 gestational weeks mediated via brainstem and are fully present at birth. Their persistence beyond 6 months of age can result in immature pattern of behavior. With maturation of central nervous system, voluntary motor activities replace the primitive reflexes. ${ }^{1}$ These primitive reflexes have been classified as normal or abnormal reflexes considering their role in growth and development. ${ }^{2}$

Reflex activity is an unconscious response to a peripheral nervous stimulation. It is a protective mechanism and it protects the body from irreparable damages. Reflex arc is the anatomical nervous pathway comprised of a receptor, afferent nerve, center, efferent nerve and an effector organ. A receptor on receiving stimuli generates the impulses in the afferent nerve which transmits sensory impulses from receptor to the center located in brain or spinal cord. The center after receiving impulses, generates appropriate motor impulses. An efferent nerve transmits motor impulse from center to effector organ to respond to

the

stimulus. ${ }^{3}$

\section{GENERAL BODY REFLEXES}

Moro reflex: It was first demonstrated by Ernst Moro. It is an involuntary response present in its complete form by 34th week (third trimester) and remains in incomplete form in premature birth. It normally disappears after 3 or 4 months. This reflex is characterized by rapid abduction and extension of arms following which both arms come close together, accompanied by crying.

Demonstrated by pulling half-way to a sitting position from supine and suddenly letting the head fall back to a short distance. Also sudden movement of neck initiates this reflex. Its absence on one side is seen in hemiplegia, injury to brachial plexus, palsy and fracture of clavicle. Its absence on both sides is seen in case of damage to the brain and spinal cord. Signs of aberrant or retained Moro reflex include chronic fatigue, constant hunger and weak immune system due to over production of stress hormones, hypersensitivity to light, sound or touch. Such children may be emotionally inappropriate, 
impulsive and/or unable to attend to tasks. This reflex helps in evaluating integration of the central nervous system (CNS). 4,5

Palmar/grasp reflex: The palmar grasp reflex appears at birth and persists until 5 or 6 months of age replaced by voluntary grasp at 45 months. It requires dual stimulus for eliciting. First by distally moving deep pressure over a specific area of the palmar surface of the hand which elicits a brief muscular contraction (the catching phase), this then develops into a strong holding phase only if traction is made on the tendons of the flexor or adductor muscles, now in contraction, the response being maintained by continued traction.

Clinical significance: Spastic form of cerebral palsy and Kernicterus show exceptionally strong grasp reflex whereas it is asymmetrical in cerebral damage and hemiplegia. ${ }^{6}$

Walking/stepping reflex: In this reflex when sole of feet touches a flat surface, one foot will be placed in front of the other and an attempt will be made to walk. It is present at birth. Because of an increased ratio of leg weight to strength it disappears at about 6 weeks and again reappears as a voluntary behavior at around 8-12 months. ${ }^{7}$

Clinical significance: The mature infants walk in heel-toe pattern whereas those who are premature have toe-heel pattern of walking.

Tonic labyrinthine reflex (TLR): It appears at birth and is fixed gradually from 6 weeks to 3 years. TLR stimulus is a change in head position (forward or backward) and response is a change in muscle tone, either flexion or extension. In flexion, the baby's legs are curled up and flexed into the fetal position. Reflex is needed to help babies through the birth canal.

Clinical significance: Retained reflex leads to spatial problems, motion sickness, poor posture, muscle tone, and visual perception difficulties. In prone position, the child is in excessive flexion and may not be able to lift or turn the head to clear the air passage. In supine position, the severely involved child is in stiff extension and cannot lift head, bring hands to midline or turn over. ${ }^{2}$
Limb placement reflex: When the front of the leg below the knee or the arm below the elbow is brought into contact with edge of the table, the child lifts the limbs over the edge. It is present at birth and disappears rapidly in early months of life.

Clinical significance: It is readily demonstrated in new born in case of neurological abnormalities and is difficult to elicit.

Parachute reflex: It is a protective reflex that appears at about 6-9 months of age \& persists thereafter. The reflex is elicited by holding the child in ventral suspension and suddenly lowering him to couch. The arms are reflexively extended forward towards couch as defensive reaction.

Clinical significance: In children with cerebral palsy, the reflex may be absent or abnormal. It would be asymmetrical in spastic hemiplegia.

Landau reflex: It is normally present from 3 months and usually disappears after 1 year of age. It is seen in vertical suspension with the head, spine and legs extended. If the head is flexed the hips, knees and the elbows also flex.

Clinical significance: Absence of this reflex occurs in hypotonia, hypertonia, and severe mental abnormality. ${ }^{8}$

\section{FACIAL REFLEXES}

Corneal reflex: The corneal reflex is also known as the blink reflex. It is an involuntary blinking of the eyelids elicited by stimulation (such as touching or a foreign body or bright light) of the cornea. It is dependent on the integrity of the $\mathrm{V}^{\text {th }}$ and the VII ${ }^{\text {th }}$ cranial nerves.

It can be elicited by distracting the patient by asking to gaze upwards. Lightly touch the cornea with a wisp of cotton wool, bringing the wool from the side of the eye. Reflex blinking of both eyes indicates normal. If the patient can feel the touch of the cotton wool (via the ophthalmic division of the trigeminal nerve) but there is no reflex blink (via facial nerve innervation of orbicularis oculi muscles) it indicates facial nerve palsy. If he/she is unable to feel touch of cotton wool this indicates trigeminal nerve palsy. ${ }^{9}$ 
Clinical significance: Helps in neurological examination, particularly when evaluating coma. Satisfactory demonstration of these reflexes helps to rule out associated nerve palsy.

Doll's eye reflex (Oculocephalic reflex): Passive turning of the head of a newborn leaves the eye behind. A distinct time lag occurs before the eyes move to a new position in keeping with the head position. It usually disappears within a week or two of birth. The oculocephalic reflex is suppressed in the vast majority of normal infants by age 11.5 weeks; failure of this reflex to appear indicates a cerebral lesion. ${ }^{10}$

Nasal reflex: Stimulation of the face or nasal cavity with water or local irritants produces apnea in neonates, breathing stops in expiration with laryngeal closure in infants, bradycardia \& lowering of cardiac output. Blood flow to skin, splanchnic area muscles \& kidney decreases, while flow to the heart \& brain remains protected. ${ }^{8}$

\section{ORAL REFLEXES}

Rooting reflex: It is present at birth. It begins at 28 weeks IU and becomes well established by $32-$ 34 weeks IU life. It disappears by 3-4 months of age. A normal response is said to occur when the cheek of a newborn touched or stroked along the side of the mouth causes him to turn the head towards the stimulated side and begins to suck.

Clinical significance: Persistence can interfere with sucking and absences is seen in neurologically impaired children. ${ }^{8}$

Sucking reflex: This is characterized by the first co-ordinated muscular activity and begins around 28 weeks of IU life and well established by 32-34 weeks. This disappears within 24 months. It is linked with the rooting reflex and breast feeding. It causes the child to suck at anything that touches the roof of their mouth. The child suddenly starts to suck simulating the way they naturally eat. It is important for meeting both psychological and nutritive needs during feeding. There are two stages to the action: Expression: When the nipple is placed between child's lips and palate, this will instinctively press it between their tongue and palate to draw out the milk. Milking: The tongue moves from areola to nipple, coaxing milk from the mother to be swallowed by the child.
Clinical Significance: Babies who are restricted from sucking due to various factors become restless and irritable. This deprivation may motivate the infant to suck finger or thumb for additional gratification.

Swallowing reflex: It begins around 12 weeks of IU life. Full swallowing reflex is established by $32-$ 36 weeks of IU life.

Infantile swallow: It is a non-conditioned congenital reflex. Until primary molar erupts, infant swallows with jaws separated and the tongue thrust forward using facial muscles.

Acquired congenital reflex: After eruption of post primary molar, from 18 months the child tends to swallow with teeth brought together by masticatory muscles, without tongue thrust.

Significance of swallowing reflex: Swallowing impairment is more common with bilateral hemispheric strokes, but can also occur with a unilateral infarction of either hemisphere. ${ }^{11}$

Gag reflex: A protective reflex seen in 18 weeks IU life. It along with reflexive pharyngeal swallowing prevents foreign body entering the throat except as part of normal swallowing and prevents choking. Touching in posterior one third region where ectoderm/ endoderm zone is present elicits a gag reflex.

Clinical significance: In damage to the Glossopharyngeal nerve, Vagus nerve, and Brain damage gag reflex is absent.

Cry reflex: It is a non-conditioned reflex seen as early as 21-29 weeks of IU life. ${ }^{9}$

Clinical significance: First verbal communication of infant which signifies as message of urgency or distress. The reflex may be absent in an infant born prematurely or in poor health.

\section{CONCLUSION}

Many neurological conditions show abnormal movement patterns in affected individuals. Infantile reflexes help to understand almost all movement disturbances that have gone astray i.e. some reflexes are not inhibited at the appropriate 
age in development whereas others fail to appear when they should. Individuals with cerebral palsy may retain some reflexes and certain primitive reflexes may reappear in adults. Identification of aberrant reflexive pattern during infancy can aid in early diagnosis of neurological conditions.

The American Academy of Pediatric Dentistry recommends that a child should visit a dentist within six months of first tooth eruption or by age of one year. Thus, knowledge of normal development of reflexes can enable pediatric dentists to monitor the child's psychomotor development periodically. Early identification of abnormal movements or reflexes can lead to early referral, diagnosis and treatment. Further research is still deemed necessary in exploring diverse aspects of primitive reflexes and their possible clinical implications.

\section{REFERENCES}

1. Zafeiriou DI. Primitive reflexes and postural reactions in the neurodevelopmental examination. Pediatr Neurol. 2004;31(1):1-8.

2. Capute AJ, Accardo PJ, Vining EP, Rubenstein JE, Walcher JR, Harryman S et al. Primitive reflex profile. A pilot study. Phys Ther 1978;58(9):1061-5.
3. Ganong WF. Review of Medical Physiology $21^{\text {st }}$ edition, Newyork: McGraw Hill; 2003.

4. Futagi Y, Toribe Y, Suzuki Y.The Grasp Reflex and Moro Reflex in Infants: Hierarchy of Primitive Reflex Responses. Int J Pediatr 2012. Article ID 191562.

5. Lehman RK, Schor NF. Neurologic Evaluation. In: KliegmanRM,Behrman RE, Jenson HB, Stanton BF, eds. Nelson Textbook of Pediatrics. 19th ed. Philadelphia, Pa: Saunders Elsevier; 2011.

6. Schott JM, Rossor MN. The grasp and other primitive reflexes. J Neurol Neurosurg Psychiatry 2003;74:558-6o.

7. Volpe JJ. Neurological examination: normal and abnormal features. In Volpe JJ. Neurology of the Newborn. 5th ed. Philadelphia, Pa: Saunders Elsevier; 2008.

8. Tandon S. Textbook of Pedodontics. 2nd ed. New Delhi: Paras publishers; 2008.

9. Richard L, Pullen JR. Testing the Corneal reflex. Nursing. 2005; 135(11):68.

10. Snir M, Hasanreisoglu M, Goldenberg-Cohen N. Suppression of the oculocephalic reflex (doll's eyes phenomenon) in normal full-term babies. Curr Eye Res. 2010;35(5):370-4.

11. Miller AJ. The neurobiology of swallowing and dysphagia. Developmental disabilities research reviews.2008; 14: 77 - 86.

\section{$\frac{\text { AUTHOR AFFILIATIONS: }}{1 .}$}

\section{Assistant Professor}

2. Senior Resident

Unit of Pedodontics and Preventive Dentistry, Oral Health Sciences Centre, PGIMER, Chandigarh.

\section{Corresponding Author:}

Dr. Rahul Morankar

Room.no 202, Senior Resident

Unit of Pedodontics and Preventive Dentistry

Oral Health Sciences Centre, PGIMER

Chandigarh -16oo12

+91- 9855501651

captainrahul88@gmail.com 


\section{LEGENDS}

\begin{tabular}{|c|l|l|}
\hline 1. & Inborn or acquired & $\begin{array}{l}\text { a) Inborn reflexes (Unconditioned) } \\
\text { b) Acquired reflexes (Conditioned) }\end{array}$ \\
\hline 2. & Situation of the center & $\begin{array}{l}\text { a) Cerebellar reflexes } \\
\text { b) Cortical reflexes } \\
\text { c) Bulbar or medullary reflexes } \\
\text { d) Midbrain reflexes } \\
\text { e) Spinal reflexes }\end{array}$ \\
\hline 3. & Purpose-functional significance & $\begin{array}{l}\text { a) Protective or flexor reflexes } \\
\text { b) Antigravity or extensor reflexes }\end{array}$ \\
\hline 4. & Number of synapse & $\begin{array}{l}\text { a) Monosynaptic reflexes } \\
\text { b) Polysynaptic reflexes }\end{array}$ \\
\hline 5. & Clinical basis & $\begin{array}{l}\text { a) Superficial reflexes } \\
\text { b) Deep reflexes } \\
\text { c) Visceral reflexes } \\
\text { d) Pathologic reflexes }\end{array}$ \\
\hline
\end{tabular}

Table 1. Classification of reflexes

\begin{tabular}{|l|l|l|}
\hline 1. & General body reflexes & A. Moro reflex \\
& & B. Palmar/grasp reflex \\
& & C. Walking/stepping reflex \\
& & D. Tonic labyrinthine reflex \\
& Facial reflexes & E. Limb placement reflex \\
& F. Parachute reflex \\
& Gral reflexes & G. Landau reflex \\
& & A. Corneal reflex \\
& B. Doll's eye reflex \\
& C. Nasal reflex \\
\hline 3. & A. Rooting reflex \\
& B. Sucking reflex \\
& C. Gag reflex \\
& D. Swallowing reflex \\
& E. Cry reflex
\end{tabular}

Table 2. General body reflexes 